\title{
Smart E-Attendance System Utilizing Eigenfaces Algorithm
}

\author{
Bashra Kadhim Oleiwi ${ }^{1}$, Farah F. Alkhalid ${ }^{2}$ \\ ${ }^{1,2}$ Control and Systems Engineering Department, University of Technology, Baghdad, Iraq. \\ ${ }^{1} 60010 @ u o t e c h n o l o g y . e d u . i q,{ }^{2} 10352 @ u o t e c h n o l o g y . e d u . i q$
}

\begin{abstract}
With the development of technologies and the availability of internet in the present era, the human has been looking for ease and efficiency in running his business, the educational field has been affected by a radical revolution as a result of the introduction of advanced technology. This has led educational institutions to provide and utilize all methods of advanced technology. This paper suggests a simple way of taking students attendance in universities and schools by using face detection and recognition with the goal of checking student's attendance in the classroom automatically. This operation is done with low cost because there is no need for special hardware to install the system in the classroom. It can be applied using a computer and a camera with low effort and high efficiency. Eigenfaces algorithm is applied in the proposed system for face detection and recognition utilizing OpenCV libraries and Visual C\#.Net 2015 with Microsoft Access 2016.
\end{abstract}

Index Terms - Smart Attendance system, Face Detection, Face Recognition, Database, OpenCV, and Eigenfaces algorithm.

\section{INTRODUCTION}

In recent times, a considerable amount of research is being conducted on an automatic attendance management system in order to be implemented as an alternative to the human in various applications. An automatic student attendance system is a very important tool to save consumption time with high efficiency. There are different methods of taking attendance in each institute, like the traditional manual method of attendance mark sheet, radio-frequency identification and other biometric techniques [1], [2]. As it is well-known, many biometric techniques such as voice identification, iris recognition, fingerprint identification and face recognition are used for objective identification and verification in various fields [3], [4].

Many ideas have been proposed for the biometric attendance system. Hence, the automatic attendance system using face recognition based on principle component analysis has been proposed in [5]. This system included a database of a set of facial patterns for the individual. Automatic students' attendance system in a classroom based on facial recognition technology is provided in [6] using three essential steps: firstly, detect and extract face image and save the facial information in a "txt" file for future references. Secondly, learn and train the facial image to compute Eigen value and Eigen vector of that image. And lastly, recognize and match face images with stored face images information in "xml". A mobile attendance system with face recognition and a GPS locator has been proposed in [7]. The face recognition is based on the Local Binary Pattern Histogram algorithm and it retrieves the student's location utilizing GPS services. The authors in [8] proposed a deep convolution neural network with more than nine layers. A vehicle data set based on multiple views and the deep learning framework were applied to test the proposed 
system. Comparison results showed that the proposed system using deep convolution neural network has a better performance than traditional vehicle recognition based on machine learning which needs vehicle location and has the low accuracy of shortcomings. The researchers in [9] developed an attendance system applying a quick response Code. The suggested system speeds up the operation of taking attendance and saves the teaching time. An internet of things based web camera technique is implemented in [10]. To compute the attendance, the student image is captured in which the details are fetched from the stored information in the database. The student attendance can be updated to the database by the administrator and the database sends the attendance details to parent using mobile SMS system. This study suggests a new system of taking attendance in universities and schools utilizing the face detection and recognition technology.

In this work, an E-attendance system is proposed based on face detection and storing the information about this face, then reconizing it when detected again. This operation is made using the Eigenfaces algorithm modeled by Visual C\#.net 2015, and Microsoft Access 2016 database.

\section{THE PROPOSED E-ATTANDANCE SYSTEMS DESCRIPTION}

In this paper, an efficient automatic attendance system using face detection and recognition has been proposed. Thus, "Eigenfaces algorithm" has been applied in the suggested system for face detection and recognition utilizing "OpenCV" libraries and Visual C\#.Net 2015 [11, 12] with Microsoft Access 2016. The proposed systems will be explained in next sections as follows:

\section{A. OpenCV}

OpenCV is used in this paper with the Eigenface algorithm. OpenCV is an open source computer vision and machine learning software library. It has been built for providing a common infrastructure for computer vision applications and accelerating the use of machine perception in the commercial products. Being a licensed product by BSD, OpenCV makes it easy for businesses for utilizing and modifying the code. The library has more than 2500 optimized algorithms, which include a comprehensive set of both classic and state-of-the-art computer vision and machine learning algorithms. These algorithms can be applied for face detection and recognition, objects identification, classification of human actions in videos, tracking camera movements, tracking moving objects and extracting 3D models of objects.

\section{B. Eigenfaces Algorithm}

Eigenfaces algorithm is one of the face recognition algorithms with an accurate recognition technique. Eigenfaces algorithm deals with special section in the image that has the face information and discards the other which has no face details, the algorithm of Eigenface contains two other algorithms (face recognition algorithm and face detection algorithm). By applying Eigenfaces algorithm, the face recognition can be carried out as follows [13]:

- Obtain images of the face called as $\mathrm{I}_{1}, \mathrm{I}_{2}, \mathrm{I}_{3} \ldots$ and $\mathrm{I}_{\mathrm{m}}$ to be arranged in an $\mathrm{N} \mathrm{x} \mathrm{N}$ matrix. Thus, faces should be in the same size and center. This high-dimensional image will be transformed to low-dimensional image by transforming the vector to $\mathrm{N}_{2} \times 1$ vector. $\Gamma$ represents an $\mathrm{N}_{2} \times 1$ vector, according to an $\mathrm{N} \times \mathrm{N}$ face image $\mathrm{I}$.

- Calculate the average of face vector $\Psi$, to find out the mean of all images.

$$
\Psi=\frac{1}{M} \sum_{i=1}^{M} \Gamma 1
$$


- Subtract the mean face vector from $\mathrm{N}_{2} \times 1$ vector, which represents the difference between the mean image and the original image. The zero mean image is given by:

$$
\varphi t=\Gamma 1-\Psi
$$

- Calculate the covariance matrix C.

$$
C=\frac{1}{M} \sum_{n=1}^{W} \Phi_{n} \Phi_{n}^{T}=A A^{T}
$$

Where $\mathrm{A}=[\Phi 1 \Phi 2 \ldots \Phi \mathrm{M}]$

\section{PROPOSED SYSTEM OVERVIEW}

The flowchart that describes the operation of an e-attendance system is shown in Fig. 1. First of all, the main information of students and lecturers are entered to the database, then in the classroom, the lecturer makes his selection (Branch, Level, Subject, Date ...), the system returns the students which belong to the selected conditions, then operates a camera to recognize the students that appeare in the camera and makes a comparison with the returned response, if matched then the student is checked as an attendee, otherwise the student is absent.

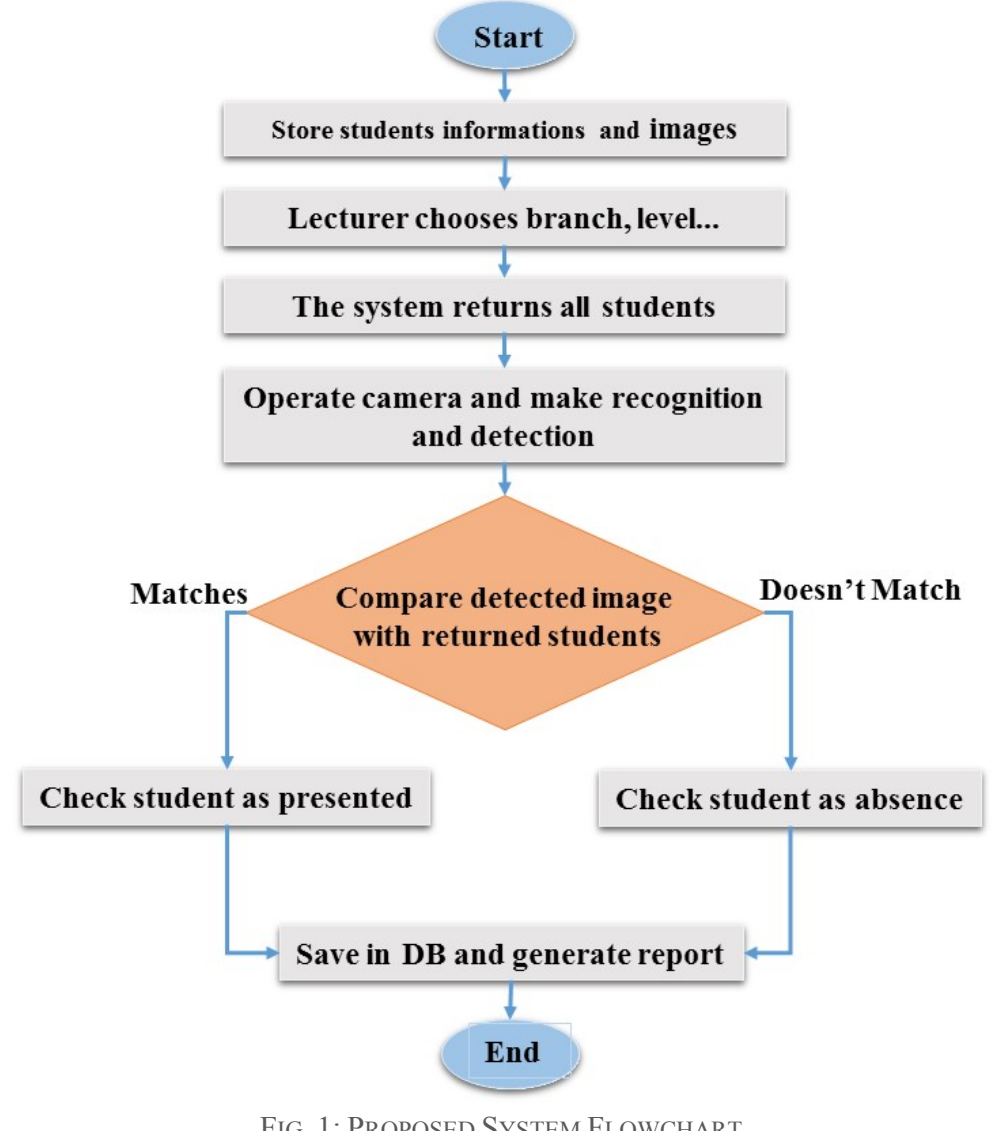

Fig. 2 shows the block diagram of the system mechanism and explains the sequence of it. 


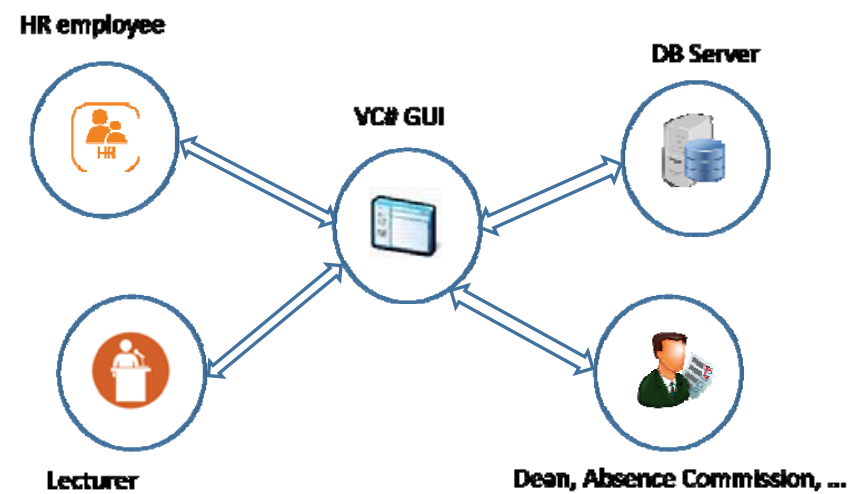

Fig. 2. BLOCK DIAGRAM OF THE PROPOSED SYSTEM.

First of all, the information of students must be entered into DB by the Human Resource (HR) employee using GUI (Graphical User Interface), the information consists of the main student's information like: (Student Name, Educational Level, Scientific section of the department, Student's image, ...), in addition, the HR employee must enter Lecturer Name, Scientific subject for each Educational Level and Scientific section.

So the lecturer will be able to choose from a drop-down list, which is shown in Fig. r, the main information like (Lecturer Name, Educational Level, Scientific Branch, Date of today (generated automatically) in order to make the attendance report more easy to generate and to avoid falling into an input writing error.

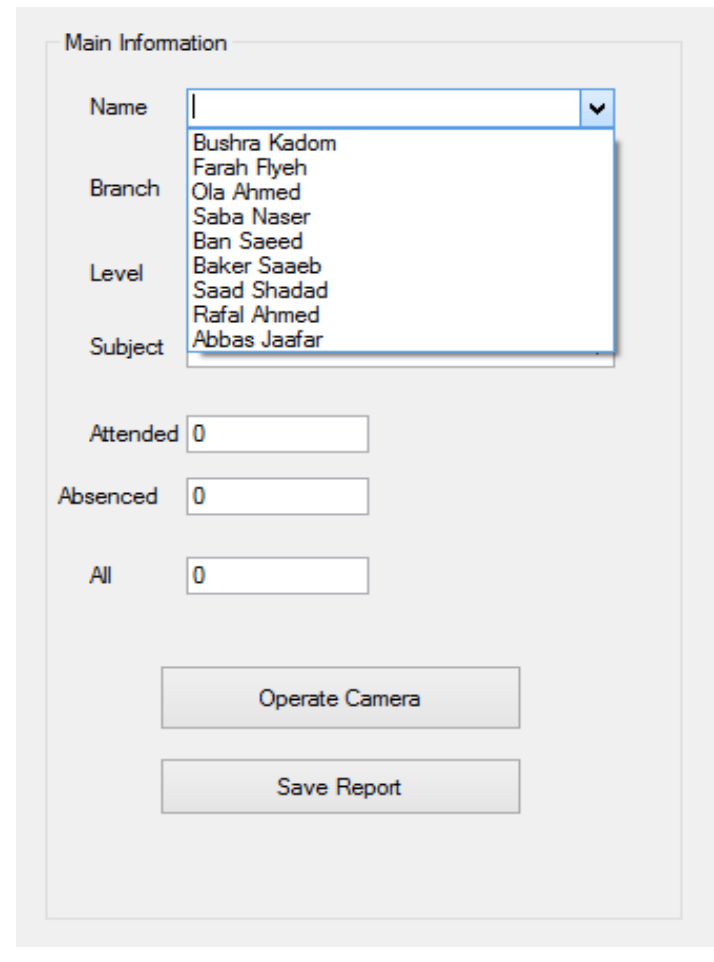

Fig. ${ }^{\Upsilon}$. SELECT INFORMATION BY LECTURER

When the lecturer finishes his lecture and fixes all the main information above, it is time to operate the camera and capture all the attended students in the class, the attendance system will recognize the students that were entered before to the $\mathrm{DB}$, and check them as attended then save them to the DB with the captured image. 
The report is ready to use by the authorized person as a reader like (Dean, Attendance Commission, ...), this report consists of the student's information in each lecture, only the lecturer can generate this report because he would be in the class and operate E-Attendance where this system would decide the student is there or not.

\section{Experimental Results}

Microsoft Visual Studio 2015 is used to program the project system using C\# language, based on Eigen algorithm to make face recognition, in addition, Microsoft Access 2016 is used as a database file to store the information of students, lecturers, subjects, and the attendance state with a profile image for each one. From the information and the image of each student entered to the DB, the Eigen algorithm in the system got only the important section in the image, which has the face features to be recognized, the captured and final images were as shown in Fig. 4.

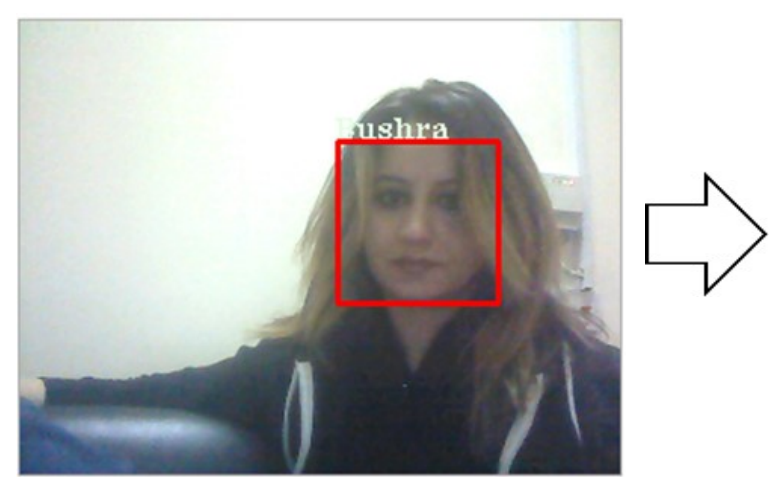

(A)

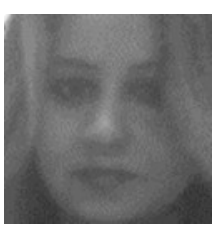

(B)

Fig. 4. (A) CAPTURED IMAgE AND (B) FinAl IMAGE AFTER USING EIgEN ALGORIthm (TEST IMAgE)

In order to verify the validity of this proposed system, a set of experiments were carried out on data obtained from cameras on the students of university and school. The Algorithm is applied on the classroom image for detection of multiple faces in the image, classroom image is processed to distinguish only the faces from the other objects present, face detection is carried out and each face is bounded by a bounding box with the student name, as demonstrated in Fig. 5. The output of the system for some different cases has been illustrated in Fig. 5, it can be seen that the results were very satisfactory as planned, where all persons were recognized successfully.

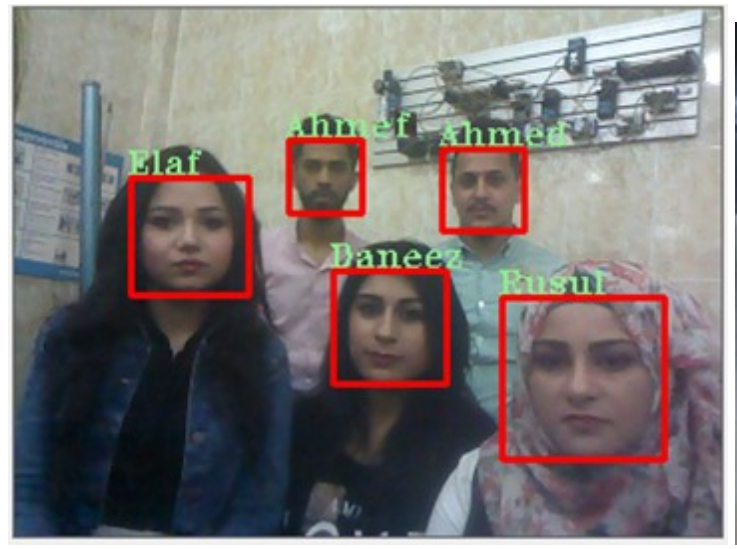

(A)

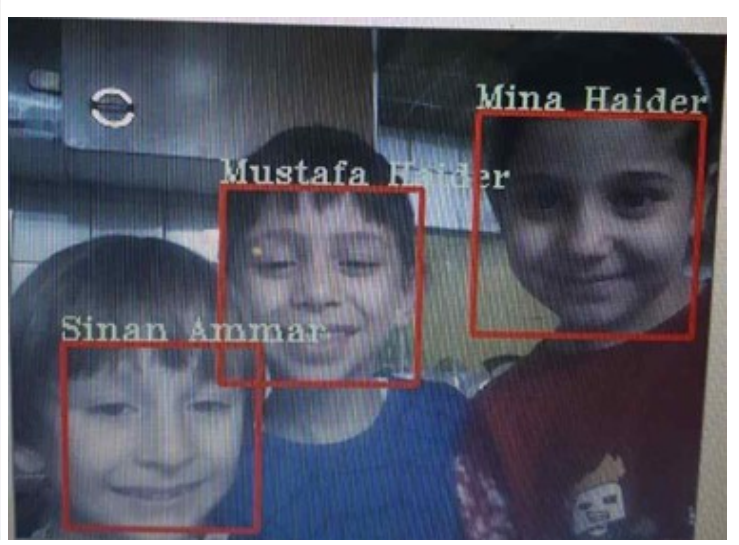

(B) 


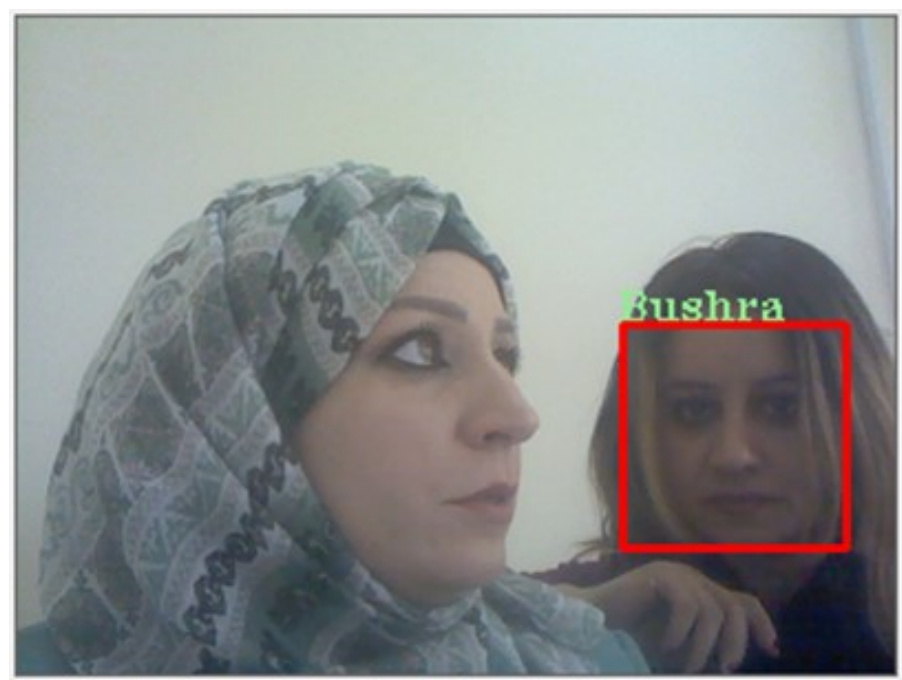

(C)

Fig. 5. Output Result. (a) And (B) Recognized and detected Persons and (c) UnRecognized Person.

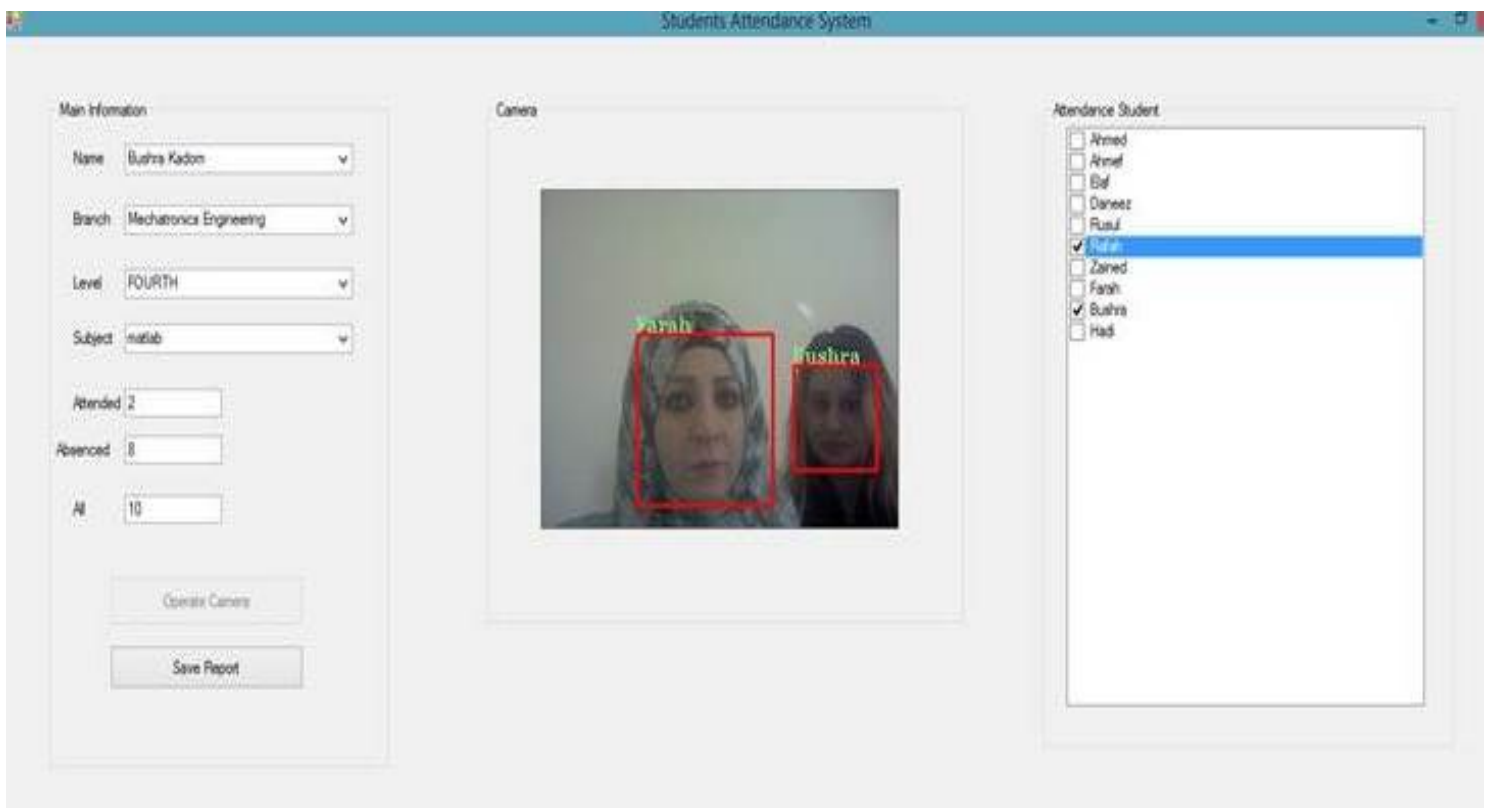

Fig. 6. GUI OF AUTOMATIC ATTENDANCE SYSTEM

The system generates a crosstab report which can be viewed by the dean or the attendance committee in order to show the students attendance state during the academic course. Fig. (7) shows the output report. 


\begin{tabular}{|l|rrrr|}
\hline & autocad & matlab & microprc & Total \\
\hline Total & 17.00 & 19.00 & 18.00 & 54.00 \\
Ahmed & 2.00 & 2.00 & 2.00 & 6.00 \\
Ahmef & 2.00 & 2.00 & 2.00 & 6.00 \\
Bushra & 2.00 & 2.00 & 2.00 & 6.00 \\
Daneez & 2.00 & 2.00 & 2.00 & 6.00 \\
Elaf & 2.00 & 2.00 & 2.00 & 6.00 \\
Farah & 2.00 & 2.00 & 2.00 & 6.00 \\
Hadi & 2.00 & 2.00 & 2.00 & 6.00 \\
Rafah & 1.00 & 2.00 & 2.00 & 5.00 \\
Rusul & 2.00 & 2.00 & 2.00 & 6.00 \\
Zained & 0.00 & 1.00 & 0.00 & 1.00 \\
\hline
\end{tabular}

Fig. 7. CRosstab Report.

The system has attained very satisfied accuracy in detecting such faces and recognizing them.

\section{CONCLUSION AND FUTURE WORK}

It can be concluded from the above experimental results that a reliable, fast, efficient and secure system has been proposed to replace a manual and unreliable system. Results have demonstrated that this system can be carried out in academic institutes to achieve better results according to attendance management. This system will reduce management efforts and save time, and will replace stationery materials with an electronic device. Hence, a system with expected results has been developed but there is still some room for improvement. The system cannot recognize a person with a very far distance of more than $10 \mathrm{~m}$ using laptop dell camera, and the students should keep calm infront of the system camera in order to make the face recognition successfully.

Finally, this proposed e-attendance system can be used in universities or schools or even in companies to make an attendance report for students or employees. This system has low cost and available requirments (copmuter with webcam, database software and executable copy of the proposed system).

In future work, this system can be modeled on microcomputer (like arduino and raspberry) to create a little kit that controls this operation. Another idea is to implement an eye of a robot to give this robot the ability to recognize people who are met before.

In addition, a new study can be conducted to make the recognition during the movement of a person.

\section{REFERENCES}

[1] G. L. Priya, M. Pandimadevi, G. R. Priya, and P. Ramya, "Implementation of attendance management system using SMART-FR", International Journal of Advance Research Computer and Communication Engineering, vol. 3, issue 11, pp. 8532-8536, November 2014

[2] M. Fuzail, H. M. F. Nouman, M. O. Mushtaq, B. R., A. Tayyab, and M. W. Talib, "face detection system for attendance of class' students", International Journal of Multidisciplinary Sciences And Engineering, vol. 5, no. 4, April 2014. 
[3] A. R. S. Siswanto, A. S. Nugroho, M. Galinium, "Implementation of face recognition algorithm for biometrics based time attendance system", International Conference on ICT For Smart Society (ICISS), Bandung, Indonesia, ISBN: 9781-4799-6322-5, 19, January 2015.

[4] A. K. Jain, A. Ross and S. Prabhakar," An introduction to biometric recognition, IEEE Transactions on Circuits and Systems for Video Technology, vol. 14, issue 1, pp. 4-20, 2004.

[5] Chitresh S. et al., "An efficient automatic attendance system using fingerprint verification technique", International Journal on Computer Science and Engineering, vol 02 , pp. 264-269, 2010.

[6] S. D. Ikhar, S. M. Bhakre, and V. M. Bodhe "A review paper on: student attendance system by face detection", International Journal of Advanced Research in Computer and Communication Engineering (JARCCE), vol. 6, Issue 1, January 2017.

[7] R. Chavan, B. Phad , S. Sawant, V. Futak, and A. Rawat," Attendance management system using face”, IJIRST International Journal for Innovative Research in Science \& Technology, vol. 1, issue 11, ISSN (online): 2349-6010, 2015.

[8] G. Baskaran, and A. F. Aznan, "Attendance system using a mobile device: Face recognition, GPS or both?", Proceedings of 41st The IRES International Conference, Prague, Czech Republic, ISBN: 978-93-86083-40-1, 23rd June 2016.

[9] X. Luoa, Ruihan Shenb, J. Huc, J. Dengd, Linji Hue and Qing Guanf, “A deep convolution neural network model for vehicle recognition and face recognition”, Xingcheng Luo et al. / Procedia Computer Science 107, 715 - 720, 2017.

[10] Md Rizal Md Hendry, M. N. A. Rahman and A.1. H. Seyal, "Smart attendance system applying QR code", 12th International Conference on Latest Trends in Engineering and Technology (ICLTET'2017) May 22-24, 2017 Kuala Lumpur, Malaysia.

[11] J. Foxall, Sams Teach Yourself Visual Basic 2015 in 24 Hours, Sams, 800 East 96th Street, Indianapolis, Indiana, 46240 USA.

[12] B. L. Jones, Sams teach yourself C\# in 21 days, Sams. 800 East 96th St., Indianapolis, Indiana, 46240 USA, 2004.

[13] Sarath Chandu Gaddam and N. V. K. Ramesh, “Attendance management and user security system's based on eigen faces algorithm using raspberry pi 2 and ethernet, Indian Journal of Science and Technology, Vol 9(17), DOI: 10.17485/ijst/2016/v9i17/92978, May 2016. 